\title{
Rate and risk factors for cataract formation and extraction after Descemet stripping endothelial keratoplasty
}

\author{
Marianne 0 Price, ${ }^{1}$ David A Price, ${ }^{1}$ Kelly M Fairchild, ${ }^{1}$ Francis W Price $\mathrm{Jr}^{2}$
}

${ }^{1}$ Cornea Research Foundation of America, Indianapolis, Indiana, USA

${ }^{2}$ Price Vision Group, Indianapolis, Indiana, USA

Correspondence to Dr Marianne 0 Price, Cornea Research Foundation of America, 9002 N. Meridian St, Suite 212, Indianapolis, IN 46260, USA;

marianneprice@cornea.org

Meeting presentation: Received best paper award at the American Academy of Ophthalmology annual meeting 26 October 2009, San Francisco, CA.

Accepted 21 January 2010 Published Online First 27 May 2010

\section{ABSTRACT}

Purpose To assess the rate and risk factors for cataract formation and extraction after Descemet stripping endothelial keratoplasty (DSEK).

Methods An initial, consecutive series of 1050 primary DSEK procedures was reviewed to identify eyes that remained phakic. Only the first-treated eye of each patient was included; 60 eyes qualified. Rate and risk factors for subsequent cataract formation and extraction were assessed by multivariate proportional hazards modelling and survival analysis.

Results Median patient age was 52 years (range: 32-69 years), and median graft diameter was $8.5 \mathrm{~mm}$ (range: $8-9 \mathrm{~mm}$ ). Median follow-up was 32 months (range: 1-51 months). Cataract extraction was performed after DSEK in 22 eyes (37\%) without complication and all grafts remained clear with median follow-up of 18 months (range: 1-44 months). Six eyes were regrafted; all underwent cataract extraction either simultaneously $(n=4)$ or subsequently $(n=2)$. At 1 and 3 years, respectively, the probability of cataract extraction was $0 \%$ and $7 \%$ in patients who were 50 years or younger at the time of DSEK $(n=20)$ versus $31 \%$ and $55 \%$ in older patients $(n=40)$, a statistically significant difference $(p=0.0005)$.

Conclusions Rates of cataract formation and extraction after DSEK were significantly higher in patients over 50 years of age and substantially exceeded normal population rates.

\section{INTRODUCTION}

Management of phakic patients with endothelial dysfunction is a common concern for corneal surgeons. In patients with visually significant lens opacification, cataract extraction is performed before keratoplasty or as a combined procedure. However, in cases with little to no lens opacification, it may be unclear whether keratoplasty alone or a triple procedure (extract, implant, keratoplasty) would better serve the patient. Previous studies have shown that $44-64 \%$ of patients develop cataracts within 5 years of penetrating keratoplasty $(\mathrm{PK})$ and that older age is a significant risk factor. ${ }^{12}$ Subsequent cataract extraction exposes patients to additional risks inherent with intraocular surgery and may increase the risk of graft failure.

Recently, endothelial keratoplasty (EK) has become the preferred treatment for endothelial dysfunction because it provides rapid visual recovery and retains the structural integrity of the eye better than standard penetrating keratoplasty (PK). ${ }^{3}{ }^{4}$ Rates of cataract formation with early EK techniques, such as posterior lamellar keratoplasty
(PLK) and deep lamellar endothelial keratoplasty (DLEK), were high, $60-100 \%$ within 2 years. ${ }^{5} 6$ Currently, the most popular form of EK is Descemet stripping endothelial keratoplasty (referred to as DSEK or DSAEK), in which dysfunctional recipient endothelium is removed and replaced with healthy donor endothelium and posterior stroma. ${ }^{4}$ Little information is available on cataract formation or cataract extraction after DSEK. Therefore, this study was undertaken to assess the rate, timing and risk factors for cataract formation and extraction after DSEK.

\section{METHODS}

\section{Participants}

A retrospective review of an initial consecutive series of 1050 primary DSEK procedures performed by a single surgeon (FWP) between December 2003 and June 2008 identified 80 eyes that remained phakic after DSEK. In bilateral cases, only the firsttreated eye of each patient was included, leaving 60 eyes for analysis. An independent review board approved the study and all patients read and signed an informed consent document. The work was compliant with the Health Insurance Portability and Accountability Act of 1996.

\section{DSEK surgical technique}

The DSEK surgical technique used in these cases was described previously, ${ }^{8}$ and a surgical video is available at http://www.aaojournal.org. In brief the donor cornea was mounted on an artificial anterior chamber, dissected manually or with a microkeratome, and cut to the desired diameter with a trephine. Dysfunctional recipient endothelium and Descemet membrane was removed and the graft was inserted through a 5-mm wide scleral tunnel or clear corneal incision using forceps $(n=57)$ or a funnel glide $(n=3)$. The graft was pressed against the recipient cornea with an air bubble, which completely filled the anterior chamber for 5-10 min. Enough air was removed to prevent pupillary block and the remaining air bubble dissipated over several days.

\section{Post-operative steroid regimen}

To prevent graft rejection, topical prednisolone acetate $1 \%$ was usually prescribed four times daily for 3-4 months, tapered over 3 months, and discontinued by $7-8$ months. Topical steroid dosing and duration was increased as needed to manage graft rejection episodes. In some cases, patients were switched to loteprednol or fluorometholone to manage intraocular pressure elevation. 


\section{Cataract formation and extraction}

Cataract formation was defined as functional disability consistent with lens opacities noted by slit lamp examination. Eighteen eyes had cataract extraction performed by one of the investigators (FWP) at the investigator's site using the soft-shell technique with $3 \%$ sodium hyaluronate $/ 4 \%$ chondroitin sulfate and $1 \%$ sodium hyaluronate viscoelastic agents. ${ }^{9}$ Cataract extraction was performed elsewhere in four eyes.

\section{Outcome measures and statistical analysis}

The primary outcome measures were rate and risk factors for subsequent cataract formation and extraction after DSEK. The statistical assessment tools were multivariate proportional hazards modelling and Kaplan-Meier survival analysis with the log rank test. These statistical methods take maximum advantage of available information when length of follow-up varies.

Endothelial cell density was measured with a Konan Noncon Robo noncontact specular microscope (Konan Medical, Hyogo, Japan), using the centre method as outlined by the manufacturer's software or with a ConfoScan 4 confocal microscope (Nidek Technologies, Greensboro, North Carolina, USA) using the manufacturer's manual cell count software. With both instruments, cell counts were obtained using the manufacturer's calibration for magnification and counts were averaged from three central photographs. In a previous study, we found no statistically significant difference between cell counts obtained with our specular and confocal microscopes.

Data were tested for normality using the KolmogorovSmirnov test. Descriptive statistics for normally distributed variables were reported as mean $\pm \mathrm{SD}$. The median and range were reported for variables that were not normally distributed. Data was analysed using SAS Version 9.0. Tests were two-sided and p-values less than 0.05 were considered statistically significant.

\section{RESULTS \\ Demographics}

The study group comprised 60 eyes (60 patients). Fifty-nine had Fuchs' endothelial dystrophy and one had unilateral endothelial decompensation and scarring of Descemet membrane without guttata or history of trauma. Forty $(60 \%)$ were female and 57 $(95 \%)$ were Caucasian. The median age was 52 years (range: 32-69 years). Two patients had non-insulin dependent diabetes mellitus.

The surgeon did not note pre-operative lens opacity in any of the study eyes during slit lamp examination. The mean preoperative central corneal thickness was $628 \pm 51 \mu \mathrm{m}$ by ultrasonic pachymetry.

The graft diameter was $9.0 \mathrm{~mm}$ in 25 eyes (42\%), $8.5 \mathrm{~mm}$ in 29 eyes (48\%), $8.25 \mathrm{~mm}$ in one eye $(1.7 \%)$, and $8.0 \mathrm{~mm}$ in five eyes (8.3\%). The median length of follow-up after DSEK was 32 months (range: $1-51$ months).

\section{Rate of cataract formation and extraction}

Cataracts formed post-operatively in 26 eyes (43\%). The probability of cataract formation was $20 \%$ at 1 year, $33 \%$ at 2 years and $44 \%$ at 3 years after DSEK (table 1). Posterior subcapsular cataract was noted in 10 eyes (17\%) after DSEK. Anterior cortical spoking was associated with cataract formation in six eyes $(10 \%)$.

Twenty-two eyes $(37 \%)$ underwent cataract extraction after DSEK. The probability of cataract extraction was $20 \%$ within 1 year, 31\% within 2 years and $40 \%$ within 3 years of DSEK (table 2). The mean age at the time of cataract extraction was $57 \pm 5.7$ years (range: $45-69$ years).
Table 1 Kaplan-Meier estimated probability of cataract developing 1 2 , and 3 years after Descemet stripping endothelial keratoplasty

\begin{tabular}{|c|c|c|c|c|c|}
\hline & Number & 1 year & 2 years & 3 years & p Value \\
\hline All & 60 & 0.20 & 0.33 & 0.44 & \\
\hline Age (years) & & & & & 0.0007 \\
\hline$\leq 50$ & 20 & 0.00 & 0.07 & 0.17 & \\
\hline$>50$ & 40 & 0.31 & 0.46 & 0.58 & \\
\hline Sex & & & & & 0.48 \\
\hline Female & 36 & 0.21 & 0.37 & 0.46 & \\
\hline Male & 24 & 0.19 & 0.31 & 0.41 & \\
\hline Graft diameter $(\mathrm{mm})$ & & & & & 0.83 \\
\hline$\leq 8.5$ & 35 & 0.23 & 0.38 & 0.44 & \\
\hline 9.0 & 25 & 0.16 & 0.31 & 0.44 & \\
\hline
\end{tabular}

\section{Risk factors}

In univariate and multivariate analyses, patient age at the time of DSEK was a significant risk factor for subsequent cataract formation and extraction. Patient sex and graft diameter were not significant risk factors (tables 1 and 2 and figure 1). Topical steroid strength, dosing frequency, and duration of use were confounded in this series, so we did not statistically assess these potential risk factors.

One graft dislocated and was successfully reattached. That eye had not developed a cataract when last examined 3 years after DSEK.

Six eyes were regrafted; all underwent cataract extraction either subsequently $(n=2)$ or simultaneously $(n=4)$. Two grafts detached, failed to clear after repositioning, and were repeated without removing the crystalline lens; cataracts formed and were extracted within a year in both eyes. The simultaneous regraft and cataract extraction group included: one eye that developed severe post-operative inflammation, one eye with narrow angles that developed intermittent graft/iris touch with intermittent graft oedema, and two eyes with visually significant wrinkles in the graft. The regrafted eyes were all in the older age group. After excluding these eyes, patient age remained a significant risk factor for cataract extraction ( $p=0.003$, table 2 ).

In 57 eyes (95\%), the graft was folded and inserted with forceps, and in three eyes (5\%) it was inserted with a funnel glide. The three funnel glide insertions all happened to be in younger recipients, who were $32-50$ years of age at the time of DSEK. All had 1-year follow-up and none developed a cataract within that time period.

\section{Complications}

No complications occurred during or after cataract extraction. Afterwards, all grafts remained clear with median follow-up of 18 months (range: $1-44$ months).

Table 2 Kaplan-Meier estimated probability of cataract extraction 1,2 , and 3 years after Descemet stripping endothelial keratoplasty

\begin{tabular}{llllll}
\hline & Number & 1 year & 2 years & 3 years & p Value \\
\hline $\begin{array}{l}\text { All } \\
\text { Age (years) }\end{array}$ & 60 & 0.20 & 0.31 & 0.40 & \\
$\quad \leq 50$ & 20 & 0.00 & 0.07 & 0.07 & 0.0005 \\
$\quad>50$ & 40 & 0.31 & 0.43 & 0.55 & \\
$\quad>50$ and no regraft & 34 & 0.20 & 0.31 & 0.46 & $0.003^{*}$ \\
Sex & & & & & 0.41 \\
$\quad$ Female & 36 & 0.21 & 0.30 & 0.43 & \\
$\quad$ Male & 24 & 0.19 & 0.31 & 0.31 & \\
Graft diameter (mm) & & & & & 0.90 \\
$\quad \leq 8.5$ & 35 & 0.23 & 0.34 & 0.38 & \\
$\quad 9.0$ & 25 & 0.16 & 0.26 & 0.40 & \\
\hline
\end{tabular}

*p value for effect of age, excluding eyes with regrafts 


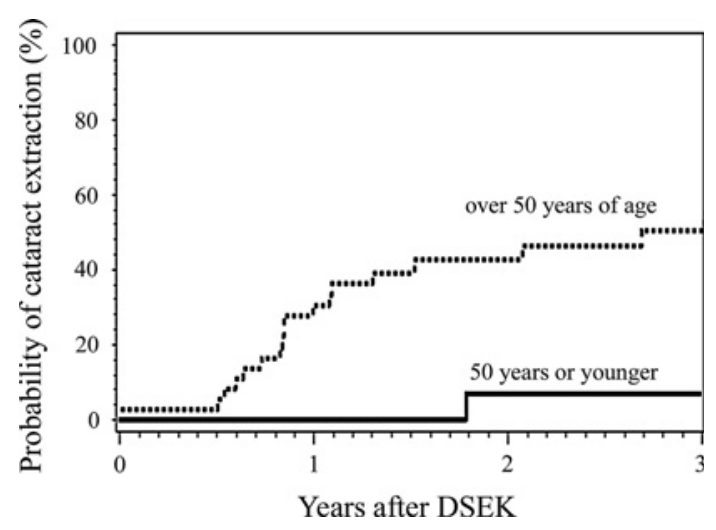

Figure 1 Kaplan-Meier estimated probability of cataract extraction after Descemet stripping endothelial keratoplasty (DSEK), segregated by patient age at the time of DSEK.

\section{Endothelial cell loss}

Endothelial cell density measurements before and after cataract extraction were available in 13 eyes. The remaining nine eyes that underwent cataract extraction either had a simultaneous regraft $(n=4)$ or the patients followed up with referring physicians $(n=5)$. The mean change in endothelial cell density after cataract extraction was $-16 \pm 144$ cells $/ \mathrm{mm}^{2} \quad(p=0.67)$. The median length of time between pre-operative and post-operative measurements was 13 months (range: 3-25 months).

\section{DISCUSSION}

This study had two key findings. First, DSEK accelerated cataract formation and cataract extraction. In American population studies, the rate of cataracts is between $3 \%$ and $4 \%$ for people in the 43-64 years age group, ${ }^{10-12}$ whereas the rate was $42 \%$ (21/ 50 eyes) in this age range in the DSEK cohort. The probability of cataract extraction was $20 \%$ within 1 year, $31 \%$ within 2 years and $40 \%$ within 3 years of DSEK. This was lower than the $60-100 \%$ rate of cataract extraction reported within 2 years of deep lamellar endothelial keratoplasty (DLEK), and comparable to rates reported after PK in eyes with Fuchs' endothelial dystrophy. ${ }^{5} 6$ Payant et al reported a cataract extraction rate of $60 \%$ with mean follow-up of 6.6 years after $\mathrm{PK},{ }^{1}$ and Martin et al found the probability of cataract extraction was $49 \%$ within 2 years and $73 \%$ within 5 years of PK in eyes with Fuchs' dystrophy. ${ }^{2}$

The second key finding was that age was a significant risk factor for cataract formation and cataract extraction after DSEK. The likelihood of cataract extraction within 3 years jumped from $7 \%$ in patients who were 50 years or younger at the time of DSEK to $55 \%$ in patients over 50 years of age. Similarly, studies have shown that age is the principal risk factor for cataract formation and cataract extraction after PK. ${ }^{12}$

Ten DSEK eyes (17\%) developed posterior subcapsular cataract. This was within the $3-32 \%$ range reported after PK. Formation of posterior subcapsular cataracts has been correlated with the dosage and duration of topical steroid use to prevent or treat immunologic graft reactions. ${ }^{13} 14$

Six DSEK eyes (10\%) developed anterior cortical spoking. Four of those were among the surgeon's first 65 DSEK cases, starting in 2003, when aspects of the DSEK technique were still early in development. The principal opportunities for intra-operative lens trauma occur during removal of dysfunctional recipient endothelium and Descemet membrane and during insertion and positioning of the graft. Two graft insertion methods were used in this study, but the number of funnel glide insertions was too few $(n=3)$ to determine if graft insertion method was a significant risk factor for cataract formation and extraction.

Cataract extraction was performed successfully after DSEK without intraoperative or post-operative complications. Cataract extraction has been associated with an increased risk of graft failure after $\mathrm{PK} .{ }^{1}{ }^{2}$ A concern specific to DSEK is that the graft reduces the space in the anterior chamber, and protrusion of the graft edge near the angle can make it difficult to avoid contact with the graft while inserting and removing phacoemulsification and irrigation and aspiration tips during cataract surgery. To minimise these difficulties, one might consider reducing the graft diameter if the crystalline lens will be left in place. Nevertheless, in this series cataract extraction was performed without intra-operative complications in 10 eyes with a $9.0-\mathrm{mm}$ graft. Furthermore, graft size did not influence the rate of cataract formation (table 1 ).

Graft central endothelial cell density did not decline significantly after cataract extraction in the subset of eyes with pre- and post-operative measurements. This is consistent with a recent PK study, in which endothelial cell loss did not differ between groups that underwent simultaneous versus sequential $\mathrm{PK}$ and cataract extraction. ${ }^{15} \mathrm{~A}$ small decline in endothelial cell density (2-3\% per year) is expected between 6 months and 3 years after DSEK. ${ }^{16}$ However, cell density measurements typically have a large SD, so a larger sample size than available in this study would be required to statistically verify the expected small decline that naturally occurs over time after DSEK.

Limitations of this study include the retrospective nature and the 32-month median length of follow-up. A prospective study with a larger number of eyes and longer follow-up would be valuable and would provide an opportunity to assess the potential relationship between pre-operative anterior chamber depth and the risk of cataract formation after DSEK. That relationship could not be assessed in this retrospective study because anterior chamber depth was not routinely measured in patients without cataract formation. Finally, while the surgeon did not note any pre-operative lens opacity in the study eyes, corneal oedema can limit the view of the lens.

This data on rate of cataract formation and extraction after DSEK can be used to counsel patients on the risks of cataract formation and aid in deciding whether to perform cataract surgery subsequent to DSEK or as part of a combined procedure. With PK, subsequent cataract extraction provides the advantage of significantly reducing mean refractive error, ${ }^{15}$ but large residual refractive errors are not a concern with DSEK. ${ }^{4}$ The obvious disadvantages of subsequent intraocular surgery are the inherent risks and cost.

In conclusion, like PK, DSEK accelerates cataract formation, particularly in patients over 50 years of age. In addition to the procedure, post-operative use of topical steroids may increase the risk of cataract formation. Subsequent cataract extraction can be performed without complications but entails additional cost and risk for the patient.

\section{Competing interests None.}

Ethics approval This study was conducted with the approval of the Institutional Review Board, Buena Park, CA.

Provenance and peer review Not commissioned; externally peer reviewed.

\section{REFERENCES}

1. Payant JA, Gordon LW, VanderZwaag R, et al. Cataract formation following corneal transplantation in eyes with Fuchs' endothelial dystrophy. Cornea 1990; 9:286-9. 
2. Martin TP, Reed JW, Legault C et al. Cataract formation and cataract extraction after penetrating keratoplasty. Ophthalmology 1994;101:113-19.

3. Melles GR, Wijdh RH, Nieuwendaal CP. A technique to excise the descemet membrane from a recipient cornea (descemetorhexis). Cornea 2004;23:286-8.

4. Price MO, Price FW. Descemet's stripping endothelial keratoplasty. Curr Opin Ophthalmol 2007:18:290-4.

5. Terry MA, Wall JM, Hoar KL, et al. A prospective study of endothelial cell loss during the 2 years after deep lamellar endothelial keratoplasty. Ophthalmology 2007:114:631-9.

6. Price MO, Price FW Jr. Cataract progression and treatment following posterior lamellar keratoplasty. J Cataract Refract Surg 2004;30:1310-15.

7. Gorovoy MS. Descemet-stripping automated endothelial keratoplasty. Cornea 2006;25:886-9

8. Price M0, Price FW Jr. Descemet's stripping with endothelial keratoplasty: comparative outcomes with microkeratome-dissected and manually dissected donor tissue. Ophthalmology 2006;113:1936-42.

9. Arshinoff SA. Dispersive-cohesive viscoelastic soft shell technique. J Cataract Refract Surg 1999;25:167-73.
10. Klein BE, Klein R, Linton KL. Prevalence of age-related lens opacities in a population. The Beaver Dam Eye Study. Ophthalmology 1992;99:546-52.

11. Leibowitz HM, Krueger DE, Maunder LR, et al. The Framingham Eye Study monograph: An ophthalmological and epidemiological study of cataract, glaucoma, diabetic retinopathy, macular degeneration, and visual acuity in a general population of 2631 adults, 1973-1975. Surv Ophthalmol 1980;24 (Suppl):335-610

12. Klein BE, Klein R. Cataracts and macular degeneration in older Americans. Arch Ophthalmol 1982:100:571-3.

13. Donshik PC, Cavanaugh HD, Boruchoff SA, et al. Posterior subcapsular cataracts induced by topical corticosteroids following keratoplasty for keratoconus. Ann Ophthalmol 1981;13:29-32

14. Rathi VM, Krishnamachary M, Gupta S. Cataract formation after penetrating keratoplasty. J Cataract Refract Surg 1997;23:562-4.

15. Hayashi $\mathbf{K}$, Hayashi $\mathrm{H}$. Simultaneous versus sequential penetrating keratoplasty and cataract surgery. Cornea 2006;25:1020-5.

16. Price FW Jr, Price MO. Does endothelial cell survival differ between DSEK and standard PK? Ophthalmology 2009;116:367-8. 